\title{
Comparing two strategies to model uncertainties in structural dynamics
}

\author{
Rubens Sampaio ${ }^{\mathrm{a}}$ and Edson Cataldo ${ }^{\mathrm{b}, *}$ \\ ${ }^{a}$ Mechanical Engineering Department, PUC-Rio, Rua Marquês de São Vicente, 225, Gávea, RJ, CEP: 22453-900, \\ Brazil \\ ${ }^{\mathrm{b}}$ Applied Mathematics Department, Graduate Program in Telecommunications Engineering, Universidade Federal \\ Fluminense, Rua Mário Santos Braga, S/N, Centro, Niterói, RJ, CEP: 24120-040, Brazil
}

Received 24 April 2008

Revised 14 January 2009

\begin{abstract}
In the modeling of dynamical systems, uncertainties are present and they must be taken into account to improve the prediction of the models. Some strategies have been used to model uncertainties and the aim of this work is to discuss two of those strategies and to compare them. This will be done using the simplest model possible: a two d.o.f. (degrees of freedom) dynamical system. A simple system is used because it is very helpful to assure a better understanding and, consequently, comparison of the strategies. The first strategy (called parametric strategy) consists in taking each spring stiffness as uncertain and a random variable is associated to each one of them. The second strategy (called nonparametric strategy) is more general and considers the whole stiffness matrix as uncertain, and associates a random matrix to it. In both cases, the probability density functions either of the random parameters or of the random matrix are deduced from the Maximum Entropy Principle using only the available information. With this example, some important results can be discussed, which cannot be assessed when complex structures are used, as it has been done so far in the literature. One important element for the comparison of the two strategies is the analysis of the samples spaces and the how to compare them.
\end{abstract}

Keywords: Uncertainties, dynamical systems, stochastic mechanics

\section{Introduction}

Uncertainties play an important role in the description of dynamical structures and to model them is helpful to improve the credibility of a model. The study of randomness associated to mechanical systems was introduced at the beginning of the $\mathrm{XX}^{\text {th }}$ century. However, at that time, only external forces were considered as random and uncertainties related to the model were not taken into account. A good review of the random vibration history, including the study of the random uncertainties present in mechanical systems, can be found in $[13,15]$.

To take into account uncertainties, in the modeling of dynamical systems, random variables should be associated to them and probability density functions should be constructed. Then, a strategy should be chosen to construct the probability density functions and this choice is a difficult task, mainly when there are not enough experimental data available. An idea, unfortunately, widely used is to associate the gaussian distribution to all the random variables involved in the problems. However, this approach can be dangerous and it can generate results which are not physically possible. For example, a positive random variable cannot, of course, assume negative values. But, if it is modeled as if it were gaussian, it could assume negative values.

\footnotetext{
${ }^{*}$ Corresponding auhtor. Tel.: +55 21 26295483; E-mail: ecataldo@im.uff.br.
} 
In this paper an approach based on the Maximum Entropy Principle is used to construct the probability density functions of the random variables modeling the uncertainties. With this strategy, only the available information is used and among all of the possible probability density functions, the one with the maximum entropy (or uncertainty) is chosen. This avoids using information which are against the physics of the problem. The theory and some applications of this method can be found in $[10,11,19,21,22]$.

Two strategies to model uncertainties are discussed and applied to a two d.o.f. system: (1) Scalar Random Variable $(S R V)$, when uncertain scalar parameters are modeled as random variables, and (2) Matrix Random Variable (MRV), when uncertain matrices are modeled as random matrices. This second strategy was developed by C. Soize [19, $21,22]$ and is relatively new. These two strategies have been already discussed $[1,5,10,16,21,22]$ but they were not compared using a simple system, as it will be done here, and the very complexity of the problems used precluded the assessment of role of the two models. Our methodology of comparison-through a simple example where the sample spaces could be easily analysed - revealed some important new results, see Sections 6.1 and 6.2.

In the literature [1,22], SRV strategy corresponds to the parametric approach and MRV strategy corresponds to the nonparametric approach to model uncertainties in Structural Dynamics. The Stochastic Finite Element Method uses SRV strategy [6,12]. The authors decided to use the terms SRV and MRV strategy instead of parametric and nonparametric approach in an attempt to simplify the explanation. The term nonparametric used in the context of model uncertainties conflicts with the term nonparametric used in the context of probability distributions.

The simple lumped parameter system studied is used in order that the attention is directed to the modeling and not to the complexity of the system. It will be even possible to construct the corresponding sample space associated to each one of the strategies performed. With this simple system, it will be clear to conclude that the (SRV) strategy is good to model data uncertainties while the (MRV) strategy is capable of taking into account model uncertainties.

The main contributions of this paper are: (1) the parametric and nonparametric strategies are presented in a simple example, with the smallest number of parameters. The example allows the comparison between the two strategies; (2) the sample spaces corresponding to the two strategies are emphasized and used as an element of comparison. It should be observed that the two sample spaces are different and also that they are independent of coordinates (in two dimensions, i.e., $2 \times 2$ matrices, the dimension of the sample space of symmetric matrices is three), they are intrinsic to the problem; (3) the sample space of MRV contains the one of SRV. However, since one deals with approximations, some outcomes from the SRV strategy can not be predicted by the MRV strategy. This result is new and unexpected, and it is discussed in this work.

The organization of this paper is as follows. The notation used is presented in Section 2. In Section 3, the deterministic dynamical system (the mean model) used to discuss the strategies is presented. The procedure to build the corresponding stochastic problem is presented in Section 4, for both strategies (SRV and MRV), and the comparison between them is performed in Section 6. The numerical simulations and more comparisons between the two strategies are presented in Section 5. Finally, in Section 7 concluding remarks are outlined.

\section{Notation used}

The following definitions and notations are used:

(1) The deterministic spring stiffnesses are represented by lowercase letters, $k_{1}$ and $k_{2}$, and the random variables associated to them, when they are considered as uncertain, are denoted by the uppercase letters, $K_{1}$ and $K_{2}$.

(2) Deterministic vectors are represented by boldface lowercase letters (e.g. $\left.\mathbf{f}=\left(f_{1}, f_{2}\right)\right)$ and random vectors are represented by boldface upper case letters (e.g. $\mathbf{Q}=\left(Q_{1}, Q_{2}\right)$ ).

(3) The deterministic stiffness matrix is represented by the upper case letters between brackets, $[K]$, and the random matrix associated to it, when it is considered as uncertain, is represented by the boldface upper case letter between brackets, $[\mathbf{K}]$.

(4) Mean values are represented underlying the corresponding random variables (e.g. $[\underline{K}, \underline{Q})$. 


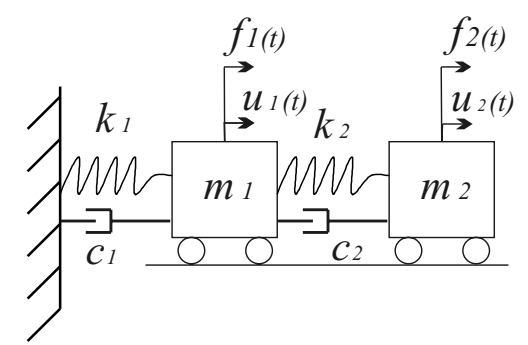

Fig. 1. Two d.o.f. system used.

\section{Mean model}

Figure 1 shows the two d.o.f. system that will be used to compare the probabilistic strategies proposed.

The equation that describe the dynamics of the system is given by:

$$
[M] \ddot{\mathbf{u}}+[C] \dot{\mathbf{u}}+[K] \mathbf{u}=\mathbf{f}(t)
$$

with

$$
\begin{aligned}
& {[M]=\left[\begin{array}{cc}
m_{1} & 0 \\
0 & m_{2}
\end{array}\right] ; \quad[C]=\left[\begin{array}{cc}
c_{1}+c_{2}-c_{2} \\
-c_{2} & c_{2}
\end{array}\right] ;} \\
& {[K]=\left[\begin{array}{cc}
k_{1}+k_{2}-k_{2} \\
-k_{2} & k_{2}
\end{array}\right]}
\end{aligned}
$$

and

$$
\mathbf{f}(t)=\left[\begin{array}{l}
f_{1}(t) \\
f_{2}(t)
\end{array}\right] ; \quad \mathbf{u}(t)=\left[\begin{array}{l}
u_{1}(t) \\
u_{2}(t)
\end{array}\right]
$$

The mass, damping, and stiffness matrices, denoted by $[M],[C]$, and $[K]$, are real symmetric and positive-definite. The external force is represented by the vector $\mathbf{f}=\left(f_{1}, f_{2}\right)^{T}$, where the functions $f_{1}$ and $f_{2}$ represent the forces applied on the masses $m_{1}$ and $m_{2}$. The displacements of the masses are denoted by the functions $u_{1}$ and $u_{2}$, the components of the vector $\mathbf{u}$.

\subsection{Frequency response function used}

In this work, the response to be observed and analyzed will be in the frequency domain, which gathers, in this example, all of the needed information.

Let $\mathbf{f}(\mathbf{t})=\left(f_{1}(t), 0\right)^{T}$ be the input force applied on the system, and let $\mathbf{u}(\mathbf{t})=\left(u_{1}(t), u_{2}(t)\right)^{T}$ be the corresponding output. Let $\widehat{f}_{1}$ be the Fourier transform of $f_{1}, \widehat{u}_{1}$ be the Fourier transform of $u_{1}$, and $\widehat{u}_{2}$ be the Fourier transform of $u_{2}$. In this paper, the Frequency Response Function (FRF) used is denoted by $h$ and defined by

$$
h(\omega)=\frac{\widehat{u}_{2}(\omega)}{\widehat{f}_{1}(\omega)} .
$$

Figure 2 shows the FRF of the mean model with the values $m_{1}=1.5 \mathrm{~kg}, m_{2}=0.75 \mathrm{~kg}, k_{1}=1000 \mathrm{~N} / \mathrm{m}, k_{2}=$ $150 \mathrm{~N} / \mathrm{m}, c_{1}=0.5 \mathrm{~N} . \mathrm{s} / \mathrm{m}, c_{2}=0.05, \mathrm{~N} . \mathrm{s} / \mathrm{m}$. The two peaks observed correspond to the natural frequencies of the system: $2.055 \mathrm{~Hz}$ and $4.502 \mathrm{~Hz}$. 


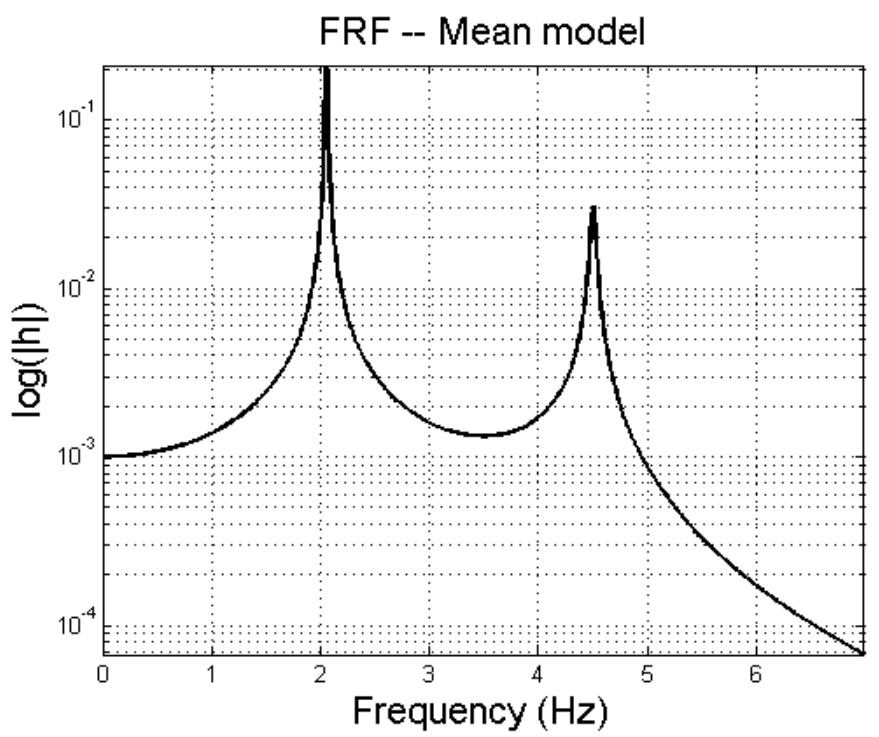

Fig. 2. FRF of the mean model.

\section{Probabilistic model}

In order to study the system behavior when uncertainties are present, some parameters or matrices must be considered as uncertain. As the main goal is to compare two different strategies to model uncertainties, only the stiffnesses will be considered as uncertain. Modeling as SRV, the parameters $k_{1}$ and $k_{2}$ are uncertain; it means that in the corresponding stochastic model these parameters will be substituted by random variables, $K_{1}$ and $K_{2}$, and for each one of these random variables a probability density function will be constructed. Modeling as MRV, the matrix $[K]$ is globally uncertain, and a probability density function will be directly associated to the corresponding random matrix, denoted by $[\mathbf{K}]$.

Clearly, for both approaches used, the stiffness matrix of the mean model should be substituted by a random matrix. Consequently, the functions $u_{1}$ and $u_{2}$, which represent the displacements of the masses, will not be deterministic anymore. Instead, they will be random processes which will be denoted by $U_{1}$ and $U_{2}$.

To construct the probability density functions, associated to the random variables $K_{1}$ and $K_{2}$ or associated to the random matrix $[\mathbf{K}]$, the Maximum Entropy Principle will be used $[8,9,11,18]$.

To construct the corresponding stochastic system, the matrix $[K]$ in Eq. (1) is substituted by the random matrix $[\mathbf{K}]$. Then, the function $\mathbf{u}$ is not deterministic anymore; it is a random process, denoted by $\mathbf{U}$. Equation (1) should be rewritten as

$$
[M] \ddot{\mathbf{U}}+[C] \dot{\mathbf{U}}+[\mathbf{K}] \mathbf{U}=\mathbf{f}(t) .
$$

It should be noted that the matrix $[\mathbf{K}]$ is constructed in a different way, depending on the probabilistic approach used, if SRV or MRV.

Let $\widehat{\mathbf{U}}=\left(\widehat{U}_{1}(\omega), \hat{U}_{2}(\omega)\right)^{T}$ and $\widehat{\mathbf{f}}(\omega)=\left(\widehat{f}_{1}(\omega), \widehat{f}_{2}(\omega)\right)^{T}$ be the Fourier transforms of $\mathbf{U}$ and $\mathbf{f}$. Then, Eq. (5) can be written, in the frequency domain, as:

$$
\left(-\omega^{2}[M]+i \omega[C]+[\mathbf{K}]\right) \widehat{\mathbf{U}}(\omega)=\widehat{\mathbf{f}}(\omega) .
$$

So, the response for the stochastic system, in the frequency domain $(\widehat{\mathbf{U}}(\omega))$ is given by

$$
\widehat{\mathbf{U}}(\omega)=\left(-\omega^{2}[M]+i \omega[C]+[\mathbf{K}]\right)^{-1} \widehat{\mathbf{f}}(\omega) .
$$

Defining $B=[0,7] \mathrm{Hz}$ as the frequency band of analysis, one has $\widehat{\mathbf{f}}(\omega)=\left(\mathbf{1}_{B}(\omega), 0\right)$, where $\mathbf{1}_{B}(\omega)=1$, if $\omega \in B$ and $\mathbf{1}_{B}(\omega)=0$, if $\omega \notin B$. 


\subsection{Maximum entropy principle}

This principle consists in maximizing the entropy (or uncertainty) subjected to constraints defined by the available information. The concept of entropy used here was that one proposed by Shannon [18].

The principle states that:

Out of all probability distributions consistent with a given set of constraints, choose the one that has maximum uncertainty (entropy).

Uncertainty can only be reduced giving additional information. It means that the probability density functions can be updated if we know more about the dynamical system we are studying. However, we must avoid using any information we are not sure about.

The measure of entropy used is an extended concept of the one used for discrete random variables and it will be defined in the following, depending if only one random variable or if a random matrix is considered.

Let $K$ be any one of the random variables $K_{1}$ or $K_{2}$ and let $p_{K}$ be the probability density function associated to $K$. Then, the entropy of $p_{K}$ is given by Eq. (8):

$$
S\left(p_{K}\right)=-\int_{-\infty}^{+\infty} p_{K}(k) \ln \left(p_{K}(k)\right) d k
$$

Let $P_{[\mathbf{K}]}$ be the probability density function associated to the random variable $[\mathbf{K}]$. Then, the entropy of $P_{[\mathbf{K}]}$ is given by:

$$
S\left(P_{[\mathbf{K}]}\right)=-\int_{\mathbb{M}_{2}^{+}(\mathbb{R})} P_{[\mathbf{K}]}([K]) \ln \left(P_{[\mathbf{K}]}([K])\right) d[K] .
$$

In both cases, the goal is to maximize the measure $S$, subject to certain constraints called available information.

According to the first part of the principle, we should use only distributions consistent with the constraints given, but there may be an infinity of them. The second part of the principle enable us to choose one out of these and we choose the one which has the maximum uncertainty for the simple reason that use of any other distribution will imply using information not given to us.

\subsection{Probabilistic model for scalar random variables (SRV)}

In this case, the parameters $k_{1}$ and $k_{2}$ are modeled by random variables $K_{1}$ and $K_{2}$. Then, an appropriate probabilistic model for each random variable must be constructed taking into account only the available information.

The available information used for both random variables $K_{1}$ and $K_{2}$ are:

(1) The random variables $K_{1}$ and $K_{2}$ are positive random variables, so their supports are equal to $] 0,+\infty[$.

(2) The expected values of $K_{1}$ and $K_{2}$ are known and given by $E\left\{K_{1}\right\}=\underline{K_{1}}$ and $E\left\{K_{2}\right\}=\underline{K_{2}}$.

(3) $K_{1}$ and $K_{2}$ are second order random variables, that is, $E\left\{K_{1}^{2}\right\}<+\infty \overline{\text { and }} E\left\{K_{2}^{2}\right\}<+\infty$.

(4) As the random variables $K_{1}$ and $K_{2}$ represent stiffnesses they must satisfy $E\left\{1 / K_{1}^{2}\right\}=c_{1}^{\prime}$, with $c_{1}^{\prime}<+\infty$, and $E\left\{1 / K_{2}^{2}\right\}=c_{2}^{\prime}$, with $c_{2}^{\prime}<+\infty$. This constraint is taken into account by requiring that $E\left\{\ln \left(K_{1}\right)\right\}=c_{1}$, with $\left|c_{1}\right|<+\infty$, and $E\left\{\ln \left(K_{2}\right)\right\}=c_{2}$, with $\left|c_{2}\right|<+\infty$.

The probability density functions obtained for $K_{1}$ and $K_{2}$ are given by:

$$
P_{K_{1}}\left(k_{1}\right)=\mathbf{1}_{] 0,+\infty[}\left(k_{1}\right) \frac{1}{\underline{K_{1}}}\left(\frac{1}{\delta_{K_{1}}^{2}}\right)^{\frac{1}{\delta_{K_{1}}^{2}}} \cdot \frac{1}{\Gamma\left(1 / \delta_{K_{1}}^{2}\right)}\left(\frac{k_{1}}{K_{1}}\right)^{\frac{1}{\delta_{K_{1}}^{2}}-1} \exp \left(-\frac{k_{1}}{\delta_{K_{1}}^{2} \underline{K_{1}}}\right)
$$

and

$$
P_{K_{2}}\left(k_{2}\right)=\mathbf{1}_{] 0,+\infty[}\left(k_{2}\right) \frac{1}{\underline{K_{2}}}\left(\frac{1}{\delta_{K_{2}}^{2}}\right)^{\frac{1}{\delta_{K_{2}}^{2}}} \cdot \frac{1}{\Gamma\left(1 / \delta_{K_{2}}^{2}\right)}\left(\frac{k_{2}}{\underline{K_{2}}}\right)^{\frac{1}{\delta_{K_{2}}^{2}}-1} \exp \left(-\frac{k_{2}}{\delta_{K_{2}}^{2} \underline{K_{2}}}\right)
$$


which are Gamma probability density functions. $\delta_{K_{1}}$ and $\delta_{K_{2}}$ are the dispersion parameters and $\Gamma$ is the Gamma function defined for $z>0$ as $\Gamma(z)=\int_{0}^{+\infty} t^{z-1} e^{-1} d t$. There are limits for the dispersion parameters: they must be greater than zero and less than $1 / \sqrt{3}$ [21].

Considering the random variables $K_{1}$ and $K_{2}$, the corresponding random matrix $[\mathbf{K}]$ becomes:

$$
[\mathbf{K}]=\left[\begin{array}{cc}
K_{1}+K_{2}-K_{2} \\
-K_{2} & K_{2}
\end{array}\right] .
$$

\subsection{Probabilistic model for matrix random variables $(M R V)$}

In this strategy, matrix $[K]$ is globally considered as uncertain, [4,7]. So, a probability density function will be constructed directly for the corresponding random matrix $[\mathbf{K}]$, which will be done according to the ideas given in [21].

In our analysis the probability distribution function for matrix $[\mathbf{K}]$ is constructed directly. But if $[\mathbf{K}]$ is derived from a Finite Element discretization a better strategy is to randomize the generalized or reduced matrix $\left[\mathbf{K}_{r}\right]$ because there might be many topological zeros in $[\underline{K}]$ and their randomization may lead to unrealistic results [20].

The following set of matrices will be considered in the text:

- $\mathbb{M}_{2}(\mathbb{R})$ is the set of all $2 \times 2$ real matrices;

- $\mathbb{M}_{2}^{S}(\mathbb{R})$ is the set of all $2 \times 2$ real symmetric matrices;

$-\mathbb{M}_{2}^{+0}(\mathbb{R})$ is the set of all $2 \times 2$ real symmetric semipositive - definite matrices;

- $\mathbb{M}_{2}^{+}(\mathbb{R})$ is the set of all $2 \times 2$ real symmetric positive - definite matrices;

- $\mathbb{M}_{2}^{D}(\mathbb{R})$ is the set of all $2 \times 2$ positive diagonal matrices.

Then, $\mathbb{M}_{2}^{D}(\mathbb{R}) \subset \mathbb{M}_{2}^{+}(\mathbb{R}) \subset \mathbb{M}_{2}^{+0}(\mathbb{R}) \subset \mathbb{M}_{2}^{S}(\mathbb{R}) \subset \mathbb{M}_{2}(\mathbb{R})$.

Let $[K]$ be a $2 \times 2$ real matrix. The Frobenius norm of $[K]$ is defined by $\|[K]\|_{F}=\left(\operatorname{tr}\left\{[K]^{T}[K]\right\}\right)^{1 / 2}$ and the matrix norm of $[K]$ is defined by $\|[K]\|=\max _{\mathbf{b} \in \mathbb{R}^{2},\|\mathbf{b}\|=1}\|[K] \mathbf{b}\|$, where $\|\mathbf{b}\|$ is the Euclidian norm of the vector $\mathbf{b}$. These norms satisfy $\|[K]\| \leqslant\|[K]\|_{F} \leqslant \sqrt{2}\|[K]\|$.

Let $[\mathbf{K}]$ be a random matrix, such that its realizations belong to $\mathbb{M}_{2}^{+}(\mathbb{R})$, and let $P_{[\mathbf{K}]}([K])$ be its probability density function. So, the normalization condition, given by Eq. (13), must be verified:

$$
\int_{\mathbb{M}_{2}^{+}(\mathbb{R})} P_{[\mathbf{K}]}([K]) d[K]=1
$$

where $d[K]=\prod_{i, j=1}^{2} d[K]_{i j}$.

The integral presented is calculated as described in the following. Let $\widetilde{\mathbf{K}}=\left(K_{11}, K_{12}, K_{21}, K_{22}\right)$ be a random vector with probability density function given by $P_{\widetilde{\mathbf{K}}}(\widetilde{\mathbf{k}})$. The probability density function of $[\mathbf{K}]$ and $\widetilde{\mathbf{K}}$ are related by:

$$
P_{\widetilde{\mathbf{K}}}(\widetilde{\mathbf{k}})=P_{[\mathbf{K}]}([K]) .
$$

Then,

$$
\int_{\mathbb{M}_{2}^{+}(\mathbb{R})} P_{[\mathbf{K}]}([K]) d[K]=\int_{\mathbb{R}^{4}} P_{\widetilde{\mathbf{K}}}(\widetilde{\mathbf{k}}) d \widetilde{\mathbf{k}}=1 .
$$


4.3.1. Using the maximum entropy principle to build the probability density function of the random matrix

In this work, a probability density function $\left(P_{[\mathbf{K}]}\right)$ of the random matrix $([\mathbf{K}])$ will be built using the Maximum Entropy Principle. The use of the Maximum Entropy for matrices was first discussed in [14] and its applications in mechanical systems was introduced in [20].

The available information used is the following:

(1) All the realizations of the random matrix $[\mathbf{K}]$ belong to the space of the $2 \times 2$ symmetric positive-definite matrices, so the support of $P_{[\mathbf{K}]}$ is the set $\mathbb{M}_{2}^{+}(\mathbb{R})$. Therefore,

$$
\int_{\mathbb{M}_{2}^{+}(\mathbb{R})} P_{[\mathbf{K}]}([K]) d[K]=1
$$

where $d[K]=\sqrt{2} \prod_{1 \leqslant i \leqslant j \leqslant 2} d[K]_{i j}=\sqrt{2} d[K]_{11} d[K]_{12} d[K]_{22}$. Note that the factor $\sqrt{2}$ appears due to the symmetry of $[\mathbf{K}]\left([\mathbf{K}]_{12}=[\mathbf{K}]_{21}\right)$.

(2) The expected value of $[\mathbf{K}]$ is known, that is:

$$
E\{[\mathbf{K}]\}=\int_{\mathbb{M}_{2}^{+}(\mathbb{R})}[K] P_{[\mathbf{K}]}([K]) d[K]=[\underline{K}] .
$$

(3) From Eq. (7), it can be proved that the corresponding random equation has a unique second-order random solution if and only if $E\left\{\left\|[\mathbf{K}]^{-1}\right\|_{F}^{2}\right\}<+\infty[21]$.

Taking into account the available information listed above and using the Maximum Entropy Principle, the probability density function of $[\mathbf{K}]$ yields [22]:

$$
P_{[\mathbf{K}]}([K])=1_{\mathbb{M}_{2}^{+}(\mathbb{R})}([K]) \times C_{\mathbf{K}} \times \operatorname{det}([K])^{\lambda-1} \times \exp \left\{\frac{-(2 \lambda+1)}{2} \operatorname{tr}[K]\right\}
$$

where

$$
C_{\mathbf{K}}=\frac{(2 \pi)^{-1 / 2}\left(\frac{1+2 \lambda}{\lambda}\right)^{1+2 \lambda}}{\Gamma\left(\frac{1+2 \lambda}{2}\right) \Gamma(\lambda)(\operatorname{det}[K])^{(1+2 \lambda) / 2}} .
$$

This probability distribution is known as the matrix Gamma variate distribution [7]. The positive real constant $\lambda$ appears due to the finiteness of the constraint $E\left\{\|\left.[\mathbf{K}]^{-1}\right|_{F} ^{2}\right\}$, and its value depends on the variance of the probabilistic model. This constant will control the dispersion of the realizations associated to the random matrix $[\mathbf{K}]$. The procedure to generate the realizations of the random matrix $[\mathbf{K}]$ will be described in the following [19-22].

Since $[\underline{K}]$ is positive-definite, using the Cholesky decomposition, one can write

$$
[\underline{\mathbf{K}}]=\left[\underline{L}_{K}\right]^{T}\left[\underline{L}_{K}\right]
$$

where $\left[L_{K}\right]$ is an upper triangular matrix.

Consequently, the random matrix $[\mathbf{K}]$ can be written as

$$
[\mathbf{K}]=\left[\underline{L}_{K}\right]^{T}[\mathbf{G}]\left[\underline{L}_{K}\right]
$$

where $[\mathbf{G}]$ is a random matrix such that:

(1) Its realizations are symmetric positive-definite matrices; (2) its mean value is the identity matrix $(E\{[\mathbf{G}]\}=$ $[I])$; and (3) the mean square value of its inverse is finite $\left(E\left\{\left\|[\mathbf{G}]^{-1}\right\|_{F}^{2}\right\}<+\infty\right)$. This constraint assures that the response of the system is a second-order random variable [21].

Taking into account the usable information and applying the Maximum Entropy Principle, the probability density function $P_{[\mathbf{G}]}$ yields:

$$
P_{[\mathbf{G}]}([G])=1_{\mathbb{M}_{2}^{+}(\mathbb{R})}([G]) \times C_{\mathbf{G}} \times \operatorname{det}([G])^{\lambda-1} \times \exp \left\{\frac{-(2 \lambda+1)}{2} \operatorname{tr}[G]\right\}
$$


where

$$
C_{\mathbf{G}}=\frac{(2 \pi)^{-1 / 2}\left(\frac{1+2 \lambda}{2}\right)^{1+2 \lambda}}{\Gamma\left(\frac{1+2 \lambda}{2}\right) \Gamma(\lambda)} .
$$

This probability distribution is also the matrix Gamma variate distribution [7]. Since the realizations of the matrix [G] are definite-positive matrices, the Cholesky decomposition can be used and one can write,

$$
[\underline{\mathbf{G}}]=[L]^{T}[L]
$$

where $[L]$ is an upper triangular matrix, given by Eq. (25).

$$
[\mathbf{L}]=\left[\begin{array}{cc}
L_{11} & L_{12} \\
0 & L_{22}
\end{array}\right] \text {. }
$$

The probability density functions of the entries of $[\mathbf{L}]\left(P_{\left[L_{11}\right]}, P_{\left[L_{12}\right]}, P_{\left[L_{22}\right]}\right)$ can be calculated using Eq. (22) and the definitions of the probability density functions of the marginal distributions. It will be concluded that:

$P_{L_{11} L_{12} L_{22}}\left(l_{11}, l_{12}, l_{22}\right)=P_{L_{11}}\left(l_{11}\right) P_{L_{12}}\left(l_{12}\right) P_{L_{22}}\left(l_{22}\right)$, i.e., the random variables $L_{11}, L_{12}$ and $L_{22}$ are statistically independents.

The constant $\lambda$ will be replaced by $\delta$, which is given by:

$$
\delta=\left\{\frac{1}{2} E\left\{\|[\mathbf{G}]-[I]\|_{F}^{2}\right\}\right\}^{\frac{1}{2}}
$$

where $0<\delta<\sqrt{\frac{3}{7}}$.

The constants $\delta$ and $\lambda$ are such that

$$
\delta=\left\{\frac{1}{1+2 \lambda}\left(1+\frac{(\operatorname{tr}[\underline{K}])^{2}}{\operatorname{tr}\left([\underline{K}]^{2}\right)}\right)\right\}^{1 / 2} .
$$

The probability density functions of the independent random variables $\left\{[\mathrm{L}]_{j j^{\prime}}, j \leqslant j^{\prime}\right\}$ are given by:

- For $j<j^{\prime}$ the real-valued random variable $[\mathrm{L}]_{j j^{\prime}}=\sigma V_{j j^{\prime}}$, in which $\sigma=\frac{\delta}{\sqrt{3}}$ and $V_{j j^{\prime}}$ is a real-valued gaussian random variable with zero mean and unit variance.

- For $j=j^{\prime}$ the real-valued random variable $[\mathrm{L}]_{j j^{\prime}}=\sigma \sqrt{2 V_{j}}$. In which $V_{j}$ is a real-valued Gamma random variable with probability density function:

$$
P_{V_{j}}(v)=\mathbf{1}_{\mathbb{R}^{+}}(v) \frac{1}{\Gamma\left(\frac{3}{2 \delta^{2}}+\frac{1-j}{2}\right)} v^{\frac{3}{2 \delta^{2}}-\frac{1+j}{2}} \exp (-v) .
$$

Then, the random matrix $[\mathbf{K}]$ becomes:

$$
[\mathbf{K}]=\left[L_{K}\right]^{T}\left[\begin{array}{cc}
2 \sigma^{2} V_{1} & \sigma^{2} \sqrt{2 V_{1}} V_{12} \\
\sigma^{2} \sqrt{2 V_{1}} V_{12} & \sigma^{2} V_{12}^{2}+2 \sigma^{2} V_{2}
\end{array}\right]\left[L_{K}\right] .
$$

Other authors are using the same strategy to construct random matrices for structural dynamical systems, see for instance Adhikari [2,3], which also discusses the selection of parameters for the MRV-strategy.

\subsection{Stochastic solver}

Let $[\mathbf{U}(\theta, \omega)]$ be the response of the stochastic system calculated for a realization $\theta$, in Eq. (7). The mean-square convergence analysis with respect to independent realizations of random variable $\widehat{\mathbf{U}}$, denoted by $\widehat{\mathbf{U}}_{j}(\theta, \omega)$, is carried out studying the function $n_{s} \mapsto \operatorname{conv}\left(n_{s}\right)$ defined by

$$
\operatorname{conv}\left(n_{s}\right)=\frac{1}{n_{s}} \sum_{j=1}^{n_{s}} \int_{B}\left\|\widehat{\mathbf{U}}_{j}(\theta, \omega)-\underline{\widehat{U}}(\omega)\right\|^{2} d \omega
$$




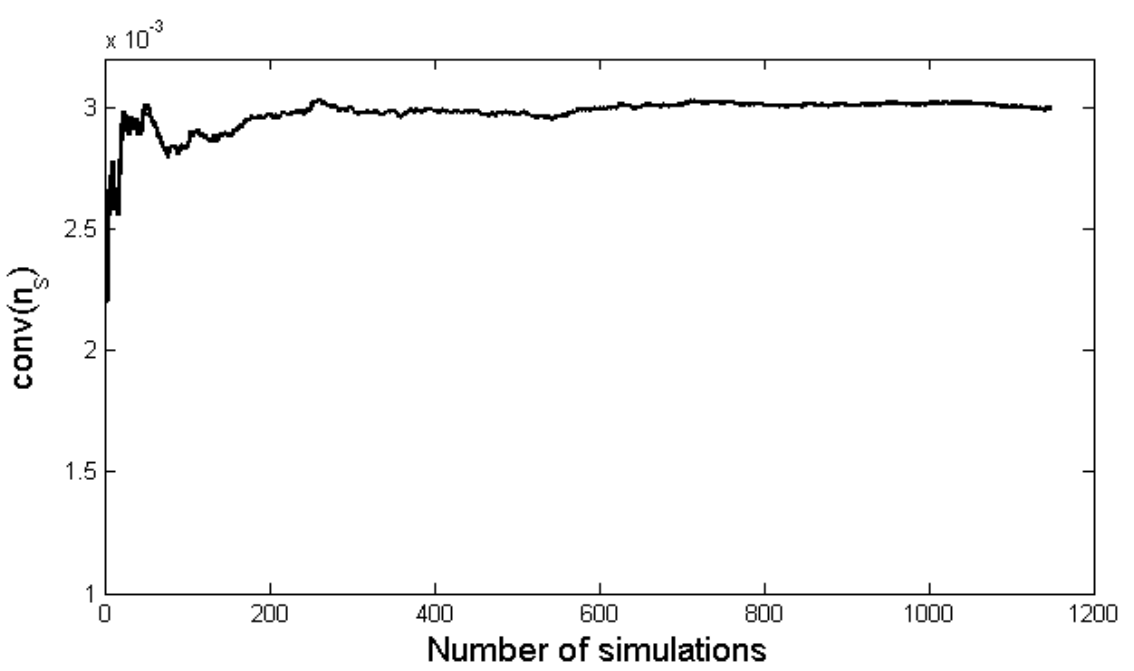

Fig. 3. Mean square convergence.

where $\widehat{\mathrm{U}}(\omega)$ is the response calculated for the corresponding mean model.

For a realization $\theta$, the $\operatorname{FRF} \mathrm{H}(\theta, \omega)$, according to Eq. (4), is given by

$$
\mathrm{H}(\theta, \omega)=\frac{\widehat{u}_{2}(\theta, \omega)}{\widehat{f}_{1}(\omega)}=\widehat{u}_{2}(\theta, \omega), \omega \in[0,7] \mathrm{Hz} .
$$

\subsection{Convergence of the solution}

To know the number of simulations $n_{s}$, Eq. (29), that assures the convergence of the approximated solution, an analysis is performed for different values of the dispersion parameter (that will be discussed later) and it is verified that for $n_{s}=600$ the approximation always converges.

Figure 3 shows an example for the function conv, considering the SRV strategy, for $\delta_{K_{1}}=\delta_{K_{2}}=0.3$. The figure shows that for $n_{s}=600$ one gets a good convergence.

Confidence regions will be constructed, for a probability level $P_{c}=0.95$, using the quantiles [17].

\section{Numerical simulations}

First of all, the same values of the coefficient of dispersion are taken for both strategies. That is: $\delta_{K_{1}}=\delta_{K_{2}}=$ $\delta_{\left[\mathbf{K}_{]}\right.}=\delta$. The coefficient $\delta$ will assume different values in order to discuss the level of dispersion associated to the problem.

Figure 4 shows the confidence region for the FRFs calculated as discussed, for $\delta=0.05,0.1,0.2$, and 0.3 .

For a low dispersion parameter, $\delta=0.05$ (Fig. 4(a)), the results are very similar for the two strategies (SRV and MRV). As the dispersion parameter increases, $\delta=0.1,0.2$, and 0.3 (Figs 4(b),(c), and (d)), the difference between the results of the two models increases as well. The response using MRV has a wider range of possible outcomes when compared with the SRV approach.

For $\delta=0.1$ the difference is still small. For $\delta=0.2$ the difference can already be noticed and for $\delta=0.3$ the difference is evident.

Figure 5 shows the FRFs when the values of the coefficients of dispersion are the greatest possible. That is, $\delta_{K_{1}}=\delta_{K_{2}}=\frac{1}{\sqrt{3}}=0.58$ and $\delta_{[\mathbf{K}]}=\sqrt{\frac{3}{7}}=0.65$.

In this case one can note that the difference between the FRFs calculated for each strategy is large. And also, the confidence region is very large, which implies that lots of solutions are inside of it. In pratical terms, this case includes, maybe, much more cases than can be obtained when experiments are performed. 


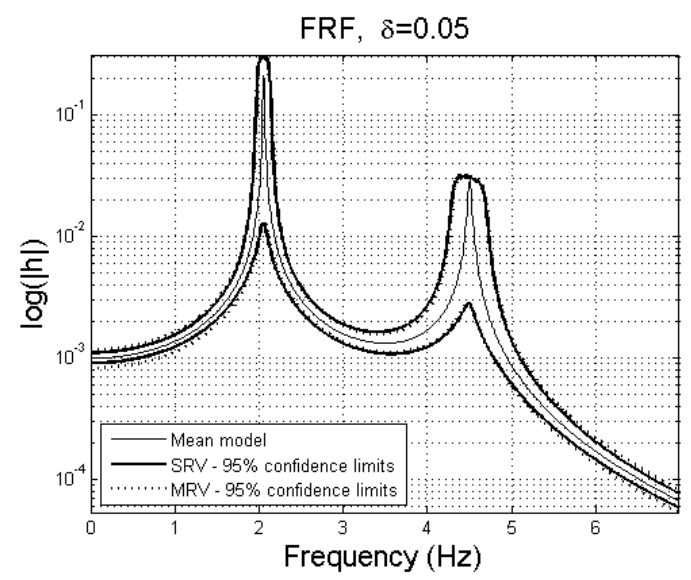

(a)

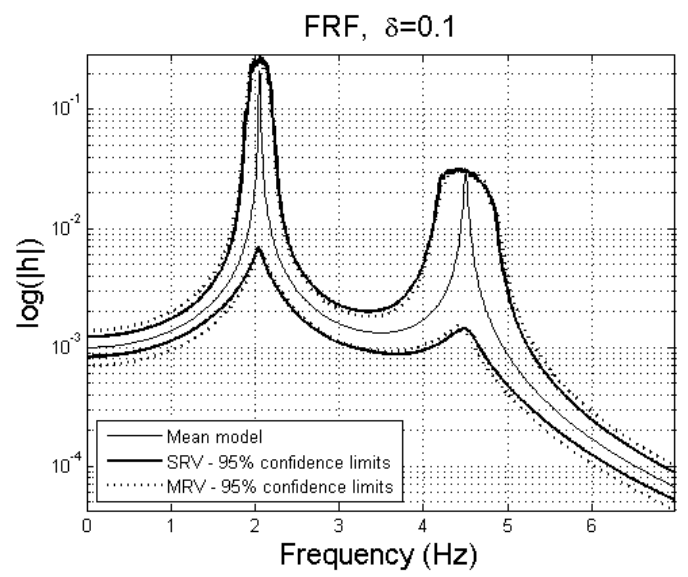

(b)

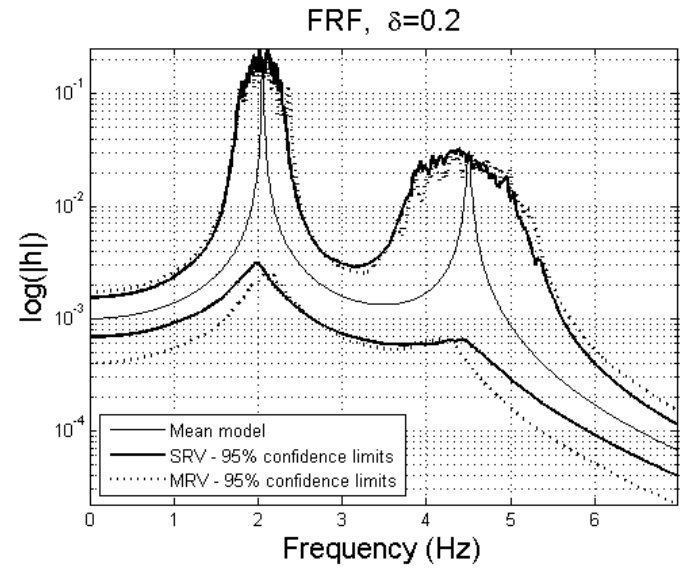

(c)

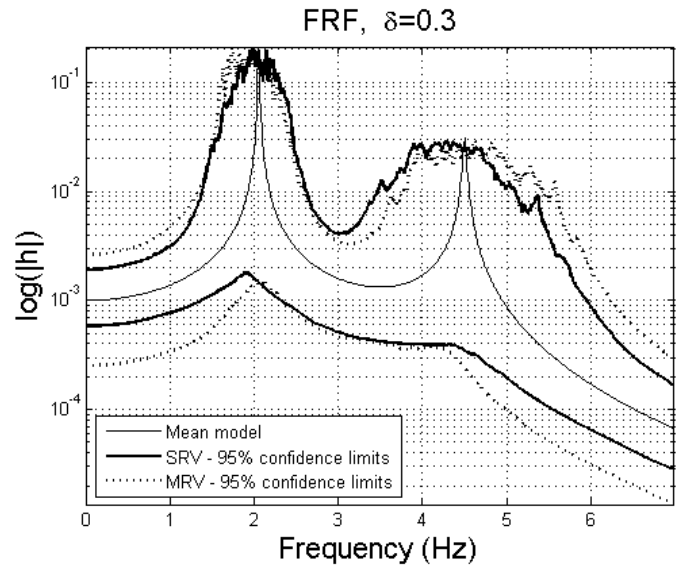

(d)

Fig. 4. Results for the FRF of the mean model with $95 \%$ confidence limits for both approaches (SRV MRV). (a) $\delta_{K_{1}}=\delta_{K_{2}}=\delta_{\left[\mathbf{K}_{]}\right.}=0.05$, (b) $\delta_{K_{1}}=\delta_{K_{2}}=\delta_{\left[\mathbf{K}_{]}\right.}=0.1$, (c) $\delta_{K_{1}}=\delta_{K_{2}}=\delta_{[\mathbf{K}]}=0.2$, (d) $\delta_{K_{1}}=\delta_{K_{2}}=\delta_{\left[\mathbf{K}_{]}\right.}=0.3$.

\section{Understanding the difference between the two strategies}

To understand why the MRV strategy leads to a wider range of possibilities, let us decouple the system by means of the modal coordinates, $\mathbf{q}=[\Phi] \mathbf{u}$, where matrix $[\Phi]$ is composed by the normalized normal modes. That is, from Eq. (1) one has:

$$
[\Phi]^{T}[M][\Phi] \ddot{\mathbf{q}}+[\Phi]^{T}[C][\Phi] \dot{\mathbf{q}}+[\Phi]^{T}[K][\Phi] \mathbf{q}=[\Phi]^{T} \mathbf{f}(t)
$$

with

$$
\begin{gathered}
{[\Phi]^{T}[M][\Phi]=\left[\begin{array}{ll}
1 & 0 \\
0 & 1
\end{array}\right] ; \quad[\Phi]^{T}[C][\Phi]=\left[\begin{array}{cc}
2 \xi_{1} \omega_{1} & 0 \\
0 & 2 \xi_{2} \omega_{2}
\end{array}\right]} \\
{[\Phi]^{T}[K][\Phi]=\left[\begin{array}{cc}
\omega_{1}^{2} & 0 \\
0 & \omega_{2}^{2}
\end{array}\right] .}
\end{gathered}
$$

Where $\omega_{1}$ and $\omega_{2}$ are the natural frequencies, and $\xi_{1}$ and $\xi_{2}(\xi=c / 2 m \omega)$ are the damping rates.

Making $\left[M_{r}\right]=[\Phi]^{T}[M][\Phi],\left[C_{r}\right]=[\Phi]^{T}[C][\Phi]$ and $\left[K_{r}\right]=[\Phi]^{T}[K][\Phi]$, Eq. (31) might be rewritten, in terms of the modal coordinates to decouple the system, by:

$$
\left[M_{r}\right] \ddot{\mathbf{q}}+\left[C_{r}\right] \dot{\mathbf{q}}+\left[K_{r}\right] \mathbf{q}=[\Phi]^{T} \mathbf{f}(t) .
$$




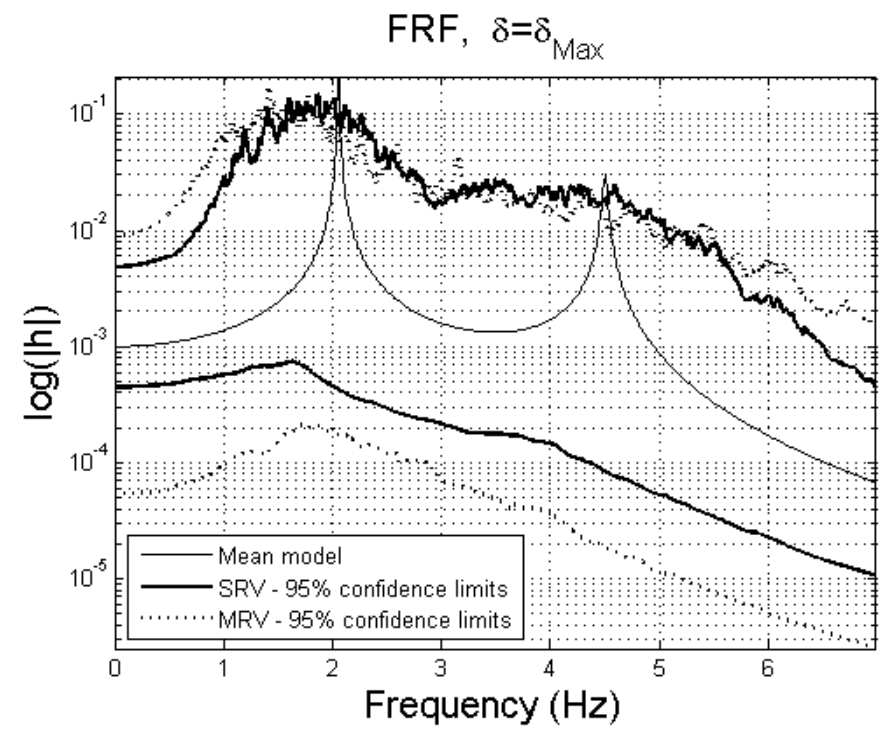

Fig. 5. Results for the FRF of the mean model with $95 \%$ confidence limits for both approaches $\left(\mathrm{SRV}\right.$ and MRV). $\delta_{K_{1}}=\delta_{K_{2}}=0.58$, $\delta_{[\mathbf{K}]}=0.65$.

Any uncertainty in $k_{1}$ or $k_{2}$ will affect $\left[K_{r}\right]$, however matrix $\left[C_{r}\right]$ will not be changed because $2 \xi \omega=c / m$.

To consider the corresponding stochastic system, the matrix $\left[K_{r}\right]$ should be substituted by $\left[\mathbf{K}_{r}\right]$.

For the mean model, one can write

$$
\left[K_{r}\right]=\left[\begin{array}{cc}
\omega_{1}^{2} & 0 \\
0 & \omega_{2}^{2}
\end{array}\right]
$$

where $\omega_{1}$ and $\omega_{2}$ are the natural frequencies of the system.

The natural frequencies will be, then, random variables given by $\Omega_{1}$ and $\Omega_{2}$. Therefore, there are two cases to be considered:

(1) In the SRV strategy, the random matrix $\left[\mathbf{K}_{r}\right]$ (denoted by $\left[\mathbf{K}_{r}\right]_{\mathrm{SRV}}$ ) is given by:

$$
\left[\mathbf{K}_{r}\right]_{\mathrm{SRV}}=\left[\begin{array}{cc}
\Omega_{1}^{2} & 0 \\
0 & \Omega_{2}^{2}
\end{array}\right] .
$$

The system remains decoupled, so the uncertainties stay within the parameters.

(2) In the MRV strategy, the random matrix $\left[\mathbf{K}_{r}\right]$ (denoted by $\left[\mathbf{K}_{r}\right]_{\mathrm{MRV}}$ ) is given by:

$$
\left[\mathbf{K}_{r}\right]_{\mathrm{MRV}}=\left[\begin{array}{cc}
\Omega_{1}^{2} & \Phi_{12} \\
\Phi_{12} & \Omega_{2}^{2}
\end{array}\right] .
$$

The random variable $\Phi_{12}$ appears due to the process of construction of $\left[\mathbf{K}_{r}\right]_{\mathrm{MRV}}$. Although the mean model is decoupled, the realizations may be coupled, because a new random variable appears in the secondary diagonal.

Typically, the study of uncertainties is divided in two parts: (1) data uncertainties, in which the values of the parameters used in the model are uncertain; and (2) model uncertainties, in which the uncertainties of the mathematical-mechanical model used for representing the real system are analyzed.

Then, according to the preceding discussion, one can conclude that the SRV strategy is helpful for modeling data uncertainties, because only the parameters of the system vary. However, using MRV strategy, it is possible to analyze uncertainties within the model, and not only data (parameters) uncertainties, because other outcomes are generated. These outcomes must have come from aspects of the problem not modeled since no variation of the parameters of the mean model can reach these new outcomes. One can note that if $\Phi_{12}=0$, then $\left[\mathbf{K}_{r}\right]_{\mathrm{MRV}}=\left[\mathbf{K}_{r}\right]_{\mathrm{SRV}}$, and any values positives of $\Omega_{1}^{2}$ and $\Omega_{2}^{2}$ are possible. However, when $\Phi_{12} \neq 0$, there is a restriction for the values of $\Omega_{1}^{2}$ and 
$\Omega_{2}^{2}$ that depend on the values of $\Phi_{12}$. One can conclude that the SRV strategy is included in the MRV strategy. In the next section we interpret the results using the sample spaces related to the strategies adopted.

The question that may remain is if all the outcomes generated are really important and to which physical system they correspond to. But, at least, we are sure that the results are coherent physically, because we assure that the realizations of $[\mathbf{K}]$ are positive definite.

\subsection{Sample spaces related to the strategies adopted}

Let $\left[\mathbf{K}_{r}(\theta)\right]$ be a realization of the random matrix $\left[\mathbf{K}_{r}\right]$. By definition, it is a positive-definite matrix. Then, one can write

$$
\left[\mathbf{K}_{r}(\theta)\right]=\left[\begin{array}{ll}
x & z \\
z & y
\end{array}\right] .
$$

Due to the construction of the stiffness matrix, one has $x>0$ and $x y-z^{2}>0$, and, consequently, $y>0$ and $z= \pm \sqrt{x y}$.

According to the SRV approach, one has $z=0$. Consequently, the sample space is two-dimensional, i.e., it is the part of the surface $z=0$ with $x>0$ and $y>0$. According to MRV approach, the sample space is three-dimensional because $z$ can now assume values from $-\sqrt{x y}$ to $+\sqrt{x y}$.

Figure 6 shows the cone generated by this condition. Figure 6(a) shows all the sample space and Fig. 6(b) shows the subset of the sample space for $z \geqslant 0$.

Note that, for $z=0$, the sample space (S) of both strategies are the same, that is, $\left.S_{S R V}\right|_{z=0}=\left.S_{M R V}\right|_{z=0}$. In general, $\mathrm{S}_{S R V} \subset \mathrm{S}_{M R V}$.

One can also note that the randomness of $\left[\mathbf{K}_{r}\right]_{\mathrm{SRV}}$ is contained in the set $\mathbb{M}_{2}^{D}(\mathbb{R})$ (positive diagonal matrices), while the randomness of $\left[\mathbf{K}_{r}\right]_{\mathrm{MRV}}$ is contained in the set $\mathbb{M}_{2}^{+}(\mathbb{R})$ (symmetric positive-definite matrices).

An important point to be noted is that, depending on the probability level considered for the confidence limits, the outcomes obtained from the SRV strategy can not be all of them inside the outcomes obtained from MRV strategy. For example, if $\delta_{K_{1}}=\delta_{K_{2}}=\delta_{[K]}$ and the probability level of $95 \%$ is taken for the confidence limits, $\left.S_{M R V}\right|_{95 \%}$ does not contain $\left.\mathrm{S}_{S R V}\right|_{95 \%}$.

For example, taking $\delta=0.2$ and the probability level $95 \%$ for the confidence limits, the realizations of matrix [K] will be given by:

$$
[\mathbf{K}(\theta)]_{\mathrm{SRV}}=\left[\begin{array}{cc}
(964 \text { to } 1362) & (-180 \text { to }-122) \\
(-180 \text { to }-122) & (122 \text { to } 180)
\end{array}\right]
$$

and

$$
[\mathbf{K}(\theta)]_{\mathrm{MRV}}=\left[\begin{array}{cc}
(974 \text { to } 1342) & (-204 \text { to }-101) \\
(-204 \text { to }-101) & (126 \text { to } 173)
\end{array}\right] .
$$

One should note that the limits of variation in the diagonal entries are greater for SRV strategy than for MRV strategy. It is clear, in this case, that $\left.S_{\mathrm{MRV}}\right|_{95 \%}$ does not contain $\left.S_{\mathrm{SRV}}\right|_{95 \%}$. This means that the parameter $\delta$ can not be directly compared.

Taking a look in the probability density functions of the first entry of each one of the matrices $[\mathbf{K}]_{\mathrm{SRV}}$ and $[\mathbf{K}]_{\mathrm{MRV}}$, one has

$$
[\mathbf{K}]_{\mathrm{SRV}}=\left[\begin{array}{cc}
K_{1}+K_{2} & \ldots \\
\cdots & \cdots
\end{array}\right]
$$

where $K_{1} \sim \operatorname{Gamma}\left(\delta_{K_{1}}^{-2}, \underline{k_{1}} \delta_{K_{1}}^{2}\right)$, and $K_{2} \sim \operatorname{Gamma}\left(\delta_{K_{2}}^{-2}, \underline{k_{2}} \delta_{K_{2}}^{2}\right)$ and 


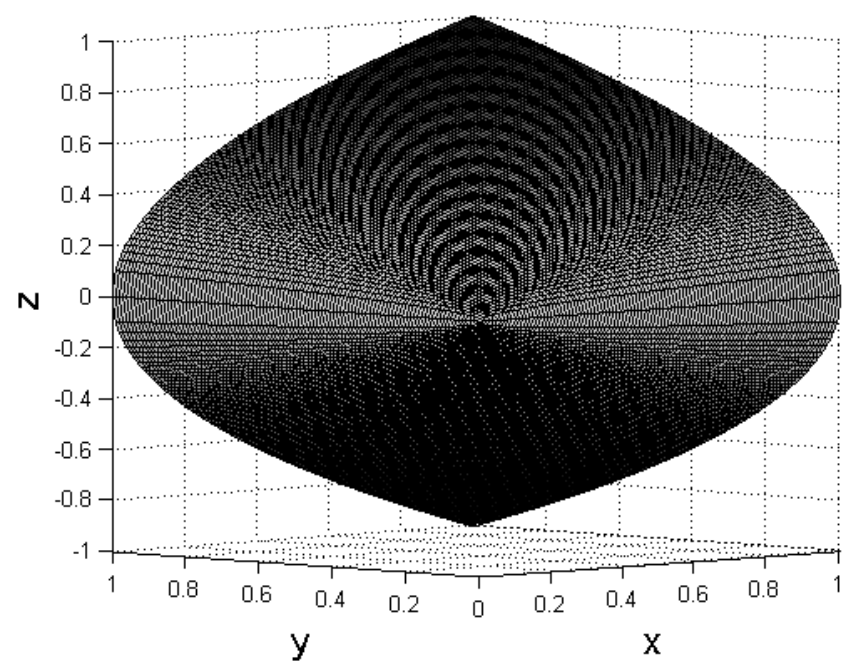

(a)

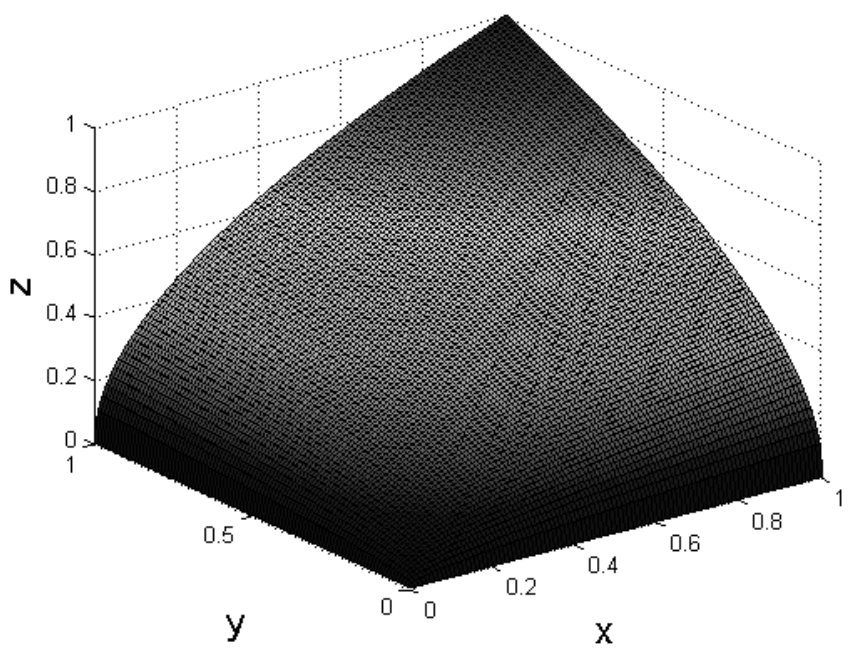

(b)

Fig. 6. Sample space for MRV strategy. (a) all the sample space and (b) sample space for $z>0$.

$$
[\mathbf{K}]_{\mathrm{MRV}}=\left[\begin{array}{cr}
\left(2 \underline{k_{1}}+2 \underline{k_{2}}\right) \sigma^{2} V_{1} \ldots \\
\cdots & \cdots
\end{array}\right]
$$

where $V_{1} \sim \operatorname{Gamma}\left(3 /\left(2 \delta_{[\mathbf{K}]}^{2}\right), 1\right)$.

Then, one can write $\left[\mathbf{K}_{\mathbf{1 1}}\right]_{\mathrm{SRV}}=K_{1}+K_{2}$ and $\left[\mathbf{K}_{\mathbf{1 1}}\right]_{\mathrm{MRV}}=\left(2 \underline{k_{1}}+2 \underline{k_{2}}\right) \sigma^{2} V_{1}$.

Figure 7 shows the probability density functions for both $\left[\mathbf{K}_{11}\right]_{\mathrm{SRV}}$ and $\left[\mathbf{K}_{11}\right]_{\mathrm{MRV}}$, with 4000 realizations performed. The mid-thick solid curve is very similar to the thin one, as it should be, because the thin curve is the analytical expression and the mid-thick curve is the result of Monte Carlo simulation for MRV strategy. The thick curve is the result of Monte Carlo simulation for SRV strategy. The dotted lines in the graphic show the 95\% confidence limits. Therefore, for the first entry of $[\mathbf{K}]$, when a confidence region is established and the $\delta$ is the same, one sees a greater possibility of outcomes for SRV strategy. 


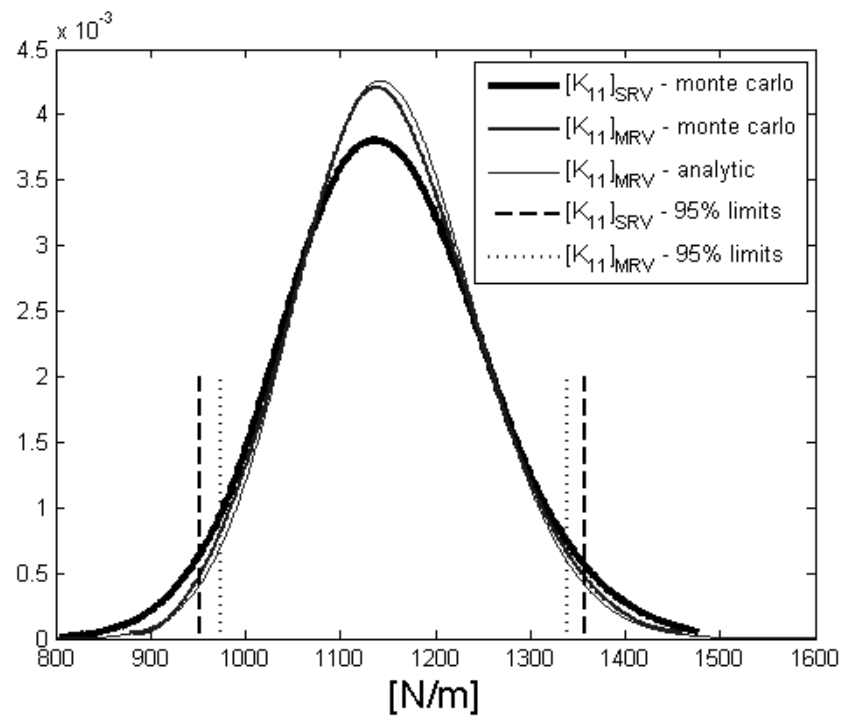

Fig. 7. Density functions of the first entry of matrix $[\mathbf{K}]$.

\subsection{Distance of the stochastic model from the mean model}

The aim here is to compare the distance of the random matrix $[\mathbf{K}]$ and the matrix $[\underline{K}]$ of the mean model using both approaches, SRV and MRV. Note that the smaller the distance the better the model. Taking an extreme, if we are sure about the mean model $[\underline{K}]$ then $\delta=0$ and we have the perfect model that corresponds perfectly to the reality (what is impossible to achieve if we are dealing with uncertain systems).

Let $[\underline{K}]$ be the stiffness matrix used in Eq. (1). Let $[\mathbf{K}]_{\mathrm{SRV}}$ be the random stiffness matrix used in Eq. (5), calculated using the SRV approach and let $[\mathbf{K}]_{\text {MRV }}$ be the random stiffness matrix used in Eq. (5), calculated using the MRV approach.

The distance of the random matrices $[\mathbf{K}]_{\mathrm{SRV}}$ and $[\underline{K}]$ will be given by:

$$
\begin{aligned}
& \operatorname{dist}_{\mathrm{SRV}}=E\left\{\left\|[\mathbf{K}]_{\mathrm{SRV}}-[\underline{K}]\right\|_{F}^{2}\right\}= \\
& \int_{\mathbb{M}_{2}^{+}(\mathbb{R})}\left\|[K]_{\mathrm{SRV}}-[\underline{K}]\right\|_{F}^{2} P_{\left[\mathbf{K}_{\mathrm{SRV}}\right.}(d[K])<+\infty
\end{aligned}
$$

and the distance of the random matrices $[\mathbf{K}]_{\mathrm{MRV}}$ and $[\underline{K}]$ will be given by:

$$
\begin{aligned}
& \operatorname{dist}_{\mathrm{MRV}}=E\left\{\left\|[\mathbf{K}]_{\mathrm{MRV}}-[\underline{K}]\right\|_{F}^{2}\right\}= \\
& \int_{\mathbb{M}_{2}^{+}(\mathbb{R})}\left\|[K]_{\mathrm{MRV}}-[\underline{K}]\right\|_{F}^{2} P_{[\mathbf{K}]_{\mathrm{MRV}}}(d[K])<+\infty .
\end{aligned}
$$

One wonders if:

$$
E\left\{\left\|[\mathbf{K}]_{\mathrm{MRV}}-[\underline{K}]\right\|_{F}^{2}\right\}<E\left\{\left\|[\mathbf{K}]_{\mathrm{SRV}}-[\underline{K}]\right\|_{F}^{2}\right\} .
$$

The values of dist $t_{\mathrm{SRV}}$ and dist $\mathrm{MRV}$ for different values of the dispersion coefficients for 1000 simulations are presented in the Table 1.

The distance increases with $\delta$, as expected, but the comparison between the two strategies is not evident. When uncertainties are taken into account, the main goal is to improve the prediction of the model. It means that one wants to minimize the distance defined by Eqs (39) and (40).

Imagine that the true $\left[K_{\exp }\right]$ (exp= experimental) is outside the parameters variation. In this case it will be possible to construct a probability density function for $[\mathbf{K}]_{\mathrm{MRV}}$ that approaches better the experiments, i.e., dist $_{\mathrm{MRV}}<$ distsRV. Note that it is likely that the true probability density function is not the one given by the 
Table 1

\begin{tabular}{ccc}
\multicolumn{4}{c}{ Table 1} \\
\hline \multicolumn{3}{c}{ Distance in function of $\delta$} \\
\hline$\delta$ & dist $_{\mathrm{SRV}}$ & dist $_{\mathrm{MRV}}$ \\
\hline 0.1 & $1.0695 \mathrm{e} 4$ & $1.0024 \mathrm{e} 4$ \\
0.2 & $4.3410 \mathrm{e} 4$ & $4.0113 \mathrm{e} 4$ \\
0.3 & $1.0525 \mathrm{e} 5$ & $8.6692 \mathrm{e} 4$ \\
0.4 & $1.8416 \mathrm{e} 5$ & $1.6487 \mathrm{e} 5$ \\
Max & $3.3605 \mathrm{e} 5$ & $4.4289 \mathrm{e} 5$ \\
\hline
\end{tabular}

Maximum Entropy Principle, but, with the information that is available, the best one can do is to use the Maximum Entropy Principle to construct the probability density function of $[\mathbf{K}]_{\mathrm{MRV}}$. On the other hand if the true $\left[K_{\exp }\right]$ is inside the parameters variations we may reduce the distance from the stochastic model to the experiments using $[\mathbf{K}]_{\mathrm{SRV}}$, i.e., dist $_{\mathrm{MRV}}>$ dist $_{\mathrm{SRV}}$.

\section{Concluding remarks}

This article presented and compared two strategies to model uncertainties in structural dynamics: the Scalar Random Variable - SRV strategy or parametric approach, in which the uncertainties are inserted into the parameters and the Matrix Random Variable-MRV strategy or nonparametric approach

Some points can also be remarked:

1. SRV and MRV approaches are different strategies to model uncertainties in a dynamical system;

2. SRV approach is good to model data uncertainties, while MRV approach is capable of taking into account model uncertainties;

3. MRV approach is able to take into account model uncertainties because some realizations of the random matrix couple the system modes (in SRV approach it does not happen);

4. The sample space resulting from SRV approach is smaller than the sample space resulting from MRV approach, what means that in MRV approach more cases might be considered;

5. It can be noted that the choice of the levels of dispersion for comparing the two approaches is not immediate.

Although both strategies are known in the literature, the discussions made here, supported by a simple example, revealed that the comparison between the two strategies is not obvious and, indeed, it may happen that they are not comparable. The main point to be emphasized is about the samples spaces: they are not the same and they have not even the same dimension. Depending on the level of uncertainties and the number of outcomes considered, the set of outcomes from the SRV strategy, whose sample space has the lowest dimension, can not be completely included in the set of outcomes of the MRV strategy. And also, the use of the strategies are directly related to the level of uncertainties considered. It should also be observed that, in pratical cases, although a large dispersion number can predict many outcomes, it is not evident that all of these outcomes are physically possible or even relevant. And, if the dispersion number is not sufficiently good, some important outcomes can not be predicted. In general, this problem is worse when the MRV strategy is used.

The use of a simple example and few parameters led us to a better understanding of both strategies, because the greater effort was not to be careful to model the dynamic of the structure, but to model the uncertainties.

\section{Acknowledgments}

This work was financed by the International Cooperation Project CAPES-COFECUB N.476/04, by CNPq (Conselho Nacional de Desenvolvimento Científico e Tecnológico), and by FAPERJ (Fundação Carlos Chagas Filho de Amparo à Pesquisa do Estado do Rio de Janeiro). 


\section{References}

[1] S. Adhikari, An unified parametric-nonparametric uncertainty quantification approach for linear dynamical systems, 48th AIAA/ASME/ASCE/AHS/ASC Structures, Structural Dynamics and Materials Conference, Waikiki, Hawaii USA, 2007.

[2] S. Adhikari, Matrix variate distributions for probabilistic structural mechanics, AIAA Journal 45(7) (2007), 1748-1762.

[3] S. Adhikari, Wishart random matrices in probabilistic structural mechanics, ASCE Journal of Engineering Mechanics 134(12) (2008), 1029-1044.

[4] P.M. Bleher, Random Matrix Models and their Applications, Cambridge University Press, England, 2001.

[5] E. Cataldo, R. Sampaio, J. Lucero and C. Soize, Modeling random uncertainties in voice production using a parametric approach, Mechanics Research Communications 35(7) (2008), 454-459.

[6] R. Ghanem and P. Spanos, Stochastic Finite Elements: A Spectral Approach, Springer-Verlag, New York, USA, 1991.

[7] A. Gupta and D. Nagar, Matrix Variate Distributions, Monographs and Surveys in Pure and Applied Mathematics, Chapman and Hall/CRC, London, England, 2000.

[8] E. Jaynes, Information theory and statistical mechanics, Phys Rev 106(4) (1957), 1620-630

[9] E. Jaynes, Information theory and statistical mechanics ii, Phys Rev 108 (1957), 171-190.

[10] E. Capiez-Lernout, M. Pellissetti, H. Pradlwarter, G.I. Schueller and C. Soize, Data and model uncertainties in complex aerospace engineering systems, Journal of Sound and Vibration 295 (2006), 923-938.

[11] J. Kapur and H. Kesavan, Entropy Optimization Principles with Applications, Academic Press, Inc., USA, 1992.

[12] M. Kleiber and T.D. Hien, The Stochastic Finite Element Method: Basic Perturbation Technique and Computer Implementation, John Wiley, Chichester, 1992.

[13] Y. Lin, Some recent advances in the theory of random vibration, Naturwissenschaften 89 (2002), 187-200.

[14] M.L. Mehta, Random Matrices, (2nd ed.), Academic Press, San Diego, CA, 1991.

[15] T. Paez, The history of random vibrations through 1958, Mechanical Systems and Signal Processing 20(8) (2006), $1783-1818$.

[16] T. Ritto, R. Sampaio and E. Cataldo, Timonshenko beam with uncertainties on the boundary conditions, Journal of the Brazilian Society of Mechanical Sciences and Engineering 30 (2008), 295-303.

[17] R.J. Serfling, Approximation Theorems of Mathematical Statistics, John Wiley and Sons, USA, 1980.

[18] C.E. Shannon, A mathematical theory of communication, Bell System Tech J 27 (1948), 379-423 and 623-659.

[19] C. Soize, A nonparametric model of random uncertainties in linear structural dynamics, Publication du LMA-CNRS, ISBN 2-909669-165152 (1999), 109-138.

[20] C. Soize, A nonparametric model of random uncertainties for reduced matrix models in structural dynamics, Probabilistic Engineering Mechanics 15(3) (2000), 277-294.

[21] C. Soize, Maximum entropy approach for modeling random uncertainties in trasient elastodynamics, Journal of the Acoustical Society of America 109(5) (2001), 1979-1996.

[22] C. Soize, A comprehensive overview of a non-parametric probabilistic approach of model uncertainties for predictive models in structural dynamics, Journal of Sound and Vibration 288(3) (2005), 623-652. 

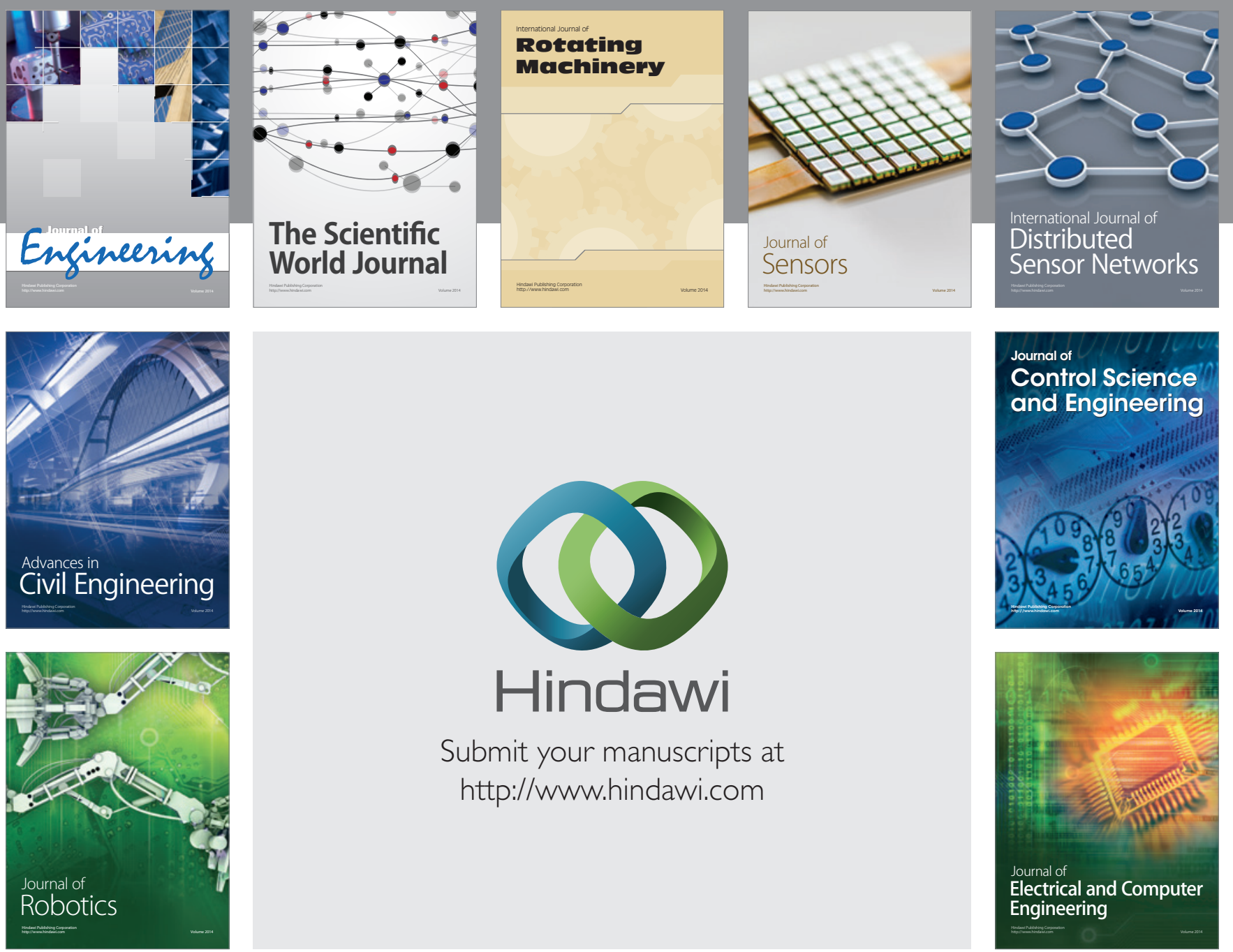

Submit your manuscripts at

http://www.hindawi.com
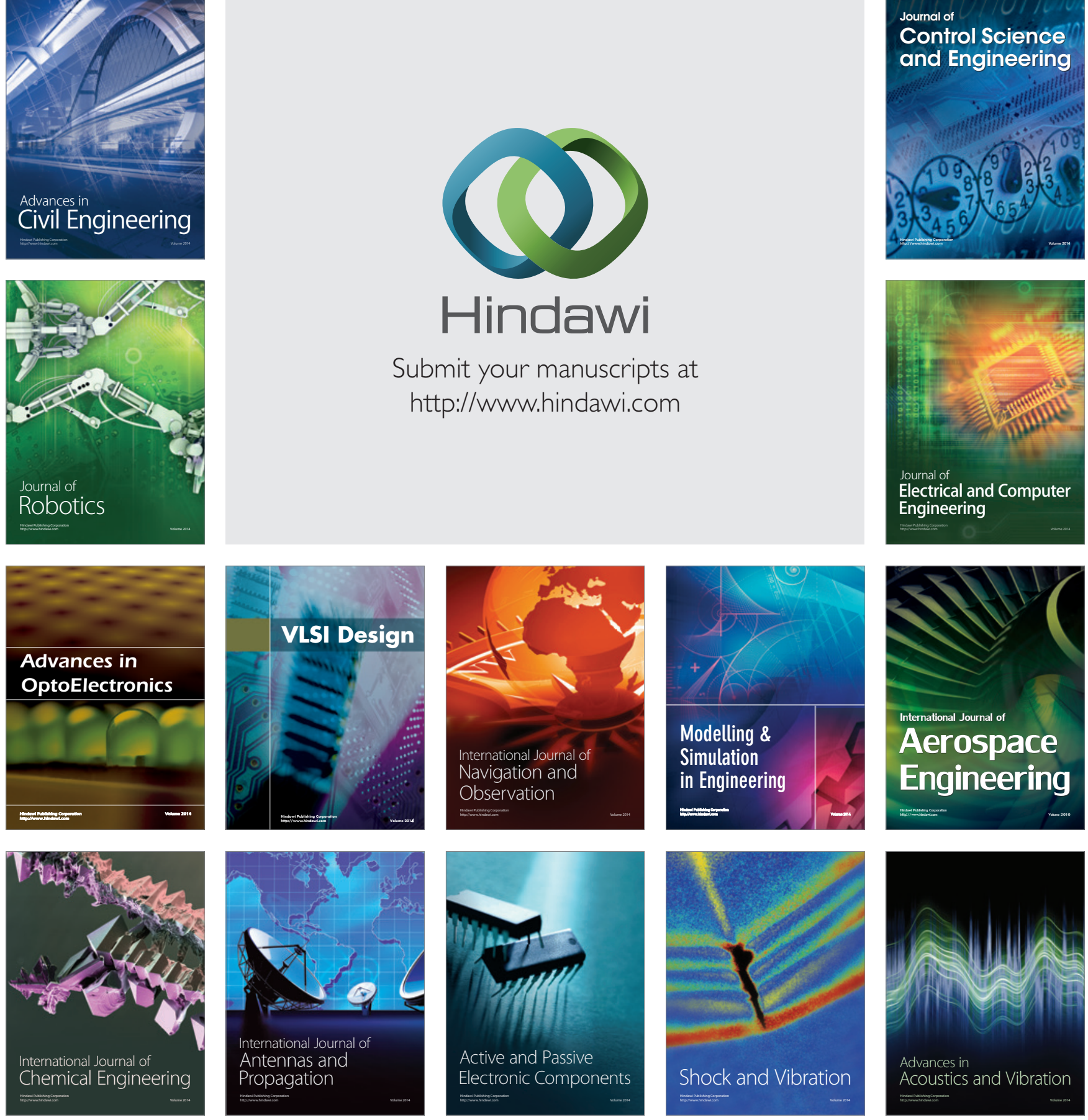